\title{
Newly defined pro-inflammatory DC subset expanded in SLE
}

Various subsets of dendritic cells (DCs) and monocytes exist that have overlapping phenotypes and functions, but delineating these subsets without ambiguity is a challenge. In a new study aimed at distinguishing monocytes from type 2 conventional DCs (cDC2s), researchers have identified a subset of cDC2s that is expanded in the blood of patients with systemic lupus erythematosus (SLE) and could be functionally involved in SLE immunopathology.

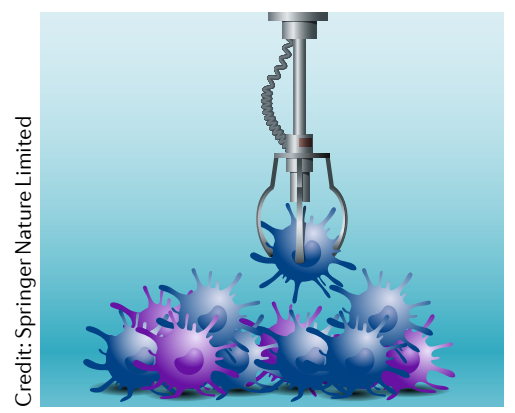

"We created a pipeline named InfinityFlow that, through machine learning-based methods, enabled us to generate high-dimensional protein expression data at the single cell level", explains Evan Newell, an author on the study. "Integrating these data allowed us to clarify grey zones in the definition of human blood mononuclear myeloid cell subsets (DCs and monocytes)".

The researchers identified markers that could distinguish monocytes (CD88 and CD89) from cDC2s (HLA-DQ and FceRIa) and further subdivide cDC2s into four phenotypically and functionally distinct subsets (on the basis of the expression of CD5, CD163 and CD14). One of the subsets, $\mathrm{CD} 5^{-} \mathrm{CD} 163^{+} \mathrm{CD} 14^{+}$cells (also referred to as inflammatory CD14 ${ }^{+}$DC3s), was increased in the blood of patients with SLE compared with the blood of patients with systemic sclerosis or healthy individuals.

Importantly, the proportion of circulating CD163+ $\mathrm{DC} 3 \mathrm{~s}$ (consisting of the aforementioned $\mathrm{CD}^{-} \mathrm{CD} 163^{+} \mathrm{CD} 14^{+}$cells and $\mathrm{CD}^{-} \mathrm{CD} 163^{+} \mathrm{CD} 14^{-}$cells) correlated with disease activity in these patients. Furthermore, RNA-sequencing analysis found that many interferon-stimulated genes, including genes encoding inflammatory cytokines, were upregulated in circulating CD163 ${ }^{+}$ DC3s from patients with SLE.

Unravelling the heterogeneity of DC subpopulations could help in the development of therapies for diseases such as SLE. "We next aim to target (eliminate or modulate) $\mathrm{CD} 14^{+}$ DC3s in SLE and several other inflammatory and autoimmune diseases," says corresponding author Florent Ginhoux.

of circulating CD 163+

DC3s ... correlated with disease activity 5

Jessica McHugh

ORIGINAL ARTICLE Dutertre, C.-A. et al. Single-cell analysis of human mononuclear phagocytes reveals subset-defining markers and identifies circulating inflammatory dendritic cells. Immunity 51, 573-589.e8 (2019) RELATED ARTICLE Cheung, P. et al. Single-cell technologies - studying rheumatic diseases one cell at a time. Nat. Rev. Rheum. 15, 340-354 (2019)

\section{Protective genes become harmful when shared via pregnancy}

Specific sequences within HLA alleles can either increase risk of or protect against rheumatoid arthritis (RA). In addition to Mendelian inheritance, HLA alleles can also be passed between mother and child during pregnancy via chimeric cells in a phenomenon known as microchimerism.

"HLA-DRB1 alleles that encode the five amino acid sequence 'DERAA' are associated with protection against RA when present in an individual's own genotype, yet in a previous study, RA prevalence was increased in women who had given birth to children with DERAA ${ }^{+}$ HLA prior to disease onset," explains Sami Kanaan, corresponding author of a new microchimerism study.

To investigate this paradox, Kanaan and colleagues measured the presence of DERAA ${ }^{+}$chimeric cells in the blood of both women with new-onset RA and healthy women, all of whom were DERAA $^{-/-}$. In this cohort, women with RA were more likely to have DERAA ${ }^{+}$ chimeric cells than healthy women. In fact, carrying DERAA ${ }^{+}$chimeric cells increased the risk of developing $R A$ by 17 -fold.

"Another previous study had reported the DERAA sequence in microbial proteins, and the presence of DERAA-directed T cells in DERAA ${ }^{-/-}$individuals that had the potential to cross-react with other synovium-expressed endogenous proteins containing DERAA, and thereby lead to RA," says Kanaan. "We reasoned that DERAA ${ }^{+}$chimeric cells could act in a similar way to microbial proteins and modelled a microchimerism scenario in vitro." In this model, DERAA ${ }^{+}$allogeneic cells elicited a response from $\mathrm{CD}^{+}$ T cells from DERAA ${ }^{-1-}$ patients with RA or healthy individuals, suggesting that chimeric cells can stimulate adaptive immune responses involved in RA.

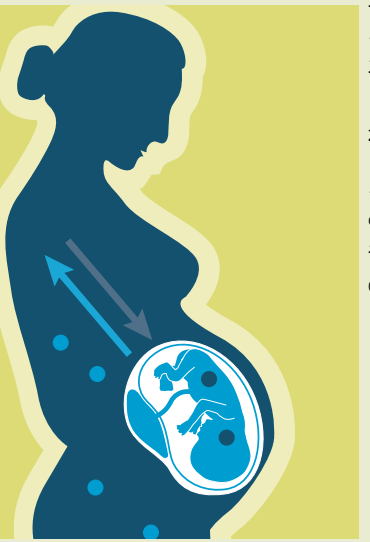

"Our study indicates a specific mechanistic link between naturally acquired allogeneic cells and an autoimmune disease for the first time, following much prior research that has implicated microchimerism in autoimmunity by association, but has offered little insight into the underlying mechanism(s)," concludes Kanaan.

Joanna Collison

ORIGINAL ARTICLE Kanaan, S. B. et al.

Immunogenicity of a rheumatoid arthritis protective sequence when acquired through microchimerism. Proc. Natl Acad. Sci. USA https://doi.org/10.1073/pnas.1904779116 (2019) 